\title{
Update on Antimicrobial Resistance and Ocular Isolates
}

\author{
Prashant Garg $\cdot$ Aravind Roy
}

Published online: 30 August 2013

(c) Springer Science + Business Media New York 2013

\begin{abstract}
The phenomenon of "Antimicrobial Resistance (AMR)" is as old as the era of antibiotics. However, currently, it is posing a serious challenge in the management of various infectious diseases including ocular infections. Realizing the seriousness of the problem, the World Health Organization and other health agencies have adopted several programs to contain the menace. The surveillance of AMR is an essential part of the action plan and has contributed significant knowledge in this field. In the last decade, three nationwide surveillance studies from United States addressed AMR among ocular isolates. These studies indicate that AMR in ocular infections shows trends similar to systemic infections. Realizing the value of surveillance studies, there is a need to conduct similar studies in other parts of the world because AMR trends demonstrate regional variations. Further, there is enough evidence to suggest that prolonged and recurrent use of topical antibiotics in ophthalmology can result in colonization of the ocular surface by resistant organisms.
\end{abstract}

Keywords Antimicrobial resistance $\cdot$ Methicillin resistance · Multi-drug resistance · Ocular TRUST · Antimicrobial resistance surveillance

\section{Introduction}

The era of antibiotics for the management of serious and often deadly infections caused by bacteria began with the

\section{P. Garg $(\bowtie) \cdot$ A. Roy}

Cornea and Anterior Segment Services, L V Prasad Eye Institute, Kallam Anji Reddy Campus, Banjara Hills, Hyderabad 500 034, India

e-mail: prashant@1vpei.org discovery of penicillin in 1928 by Alexander Fleming. The euphoria for this discovery was so much that, in 1944 when penicillin was introduced to general clinical practice, the term "Miracle Drug" entered the common vernacular. By 1965, physicians and scientists felt that bacterial diseases and the microbes responsible were no longer of great concern. Unfortunately, it did not take too long for them to realize the existence of resistant bacteria [1-3], and by 1980, fewer than $10 \%$ of all clinical Staphylococcus cases could be cured by penicillin-a dramatic shift from the almost $100 \%$ susceptibility of Staphylococcus in 1952 [4, 5]. To overcome this phenomenon of antibiotic resistance, scientists developed newer molecules with modified mechanisms of action. Little attention was paid to the seriousness and long-term consequences of Antimicrobial Resistance (AMR). Over a period of time, bacteria causing common infections have developed resistance to each new antibiotic, and today AMR has evolved to become a worldwide health threat and a critical issue $[6 \bullet \bullet, 7,8]$.

Although AMR is a public health concern primarily for diseases such as malaria, tuberculosis and to some extent $\mathrm{HIV}$, it is also becoming increasingly important for some of the common bacterial diseases [9]. Organisms such as methicillin-resistant Staphylococcus aureus (MRSA) and Coagulase Negative Staphylococci (CNS), and drug-esistant Enterobacteriaceae, as well as the emergence of multidrug-resistant bacteria, have created serious challenges to the treatment of infectious diseases including ocular infections. Moreover, the phenomenon of drug resistance is no longer restricted to hospital-acquired infections but is also being reported among community-acquired infections $[5,10]$. The situation is further complicated by the dearth of new antibiotics in the pipeline, raising the possibility that untreatable multi-drug resistant (MDR) infections will become more and more common [11, 12]. The infections 
caused by drug-resistant organisms are not only difficult to manage but are also associated with increased morbidity, mortality and duration of hospital stay, thereby resulting in substantial increases in the health care cost and financial burden to the community [13-15].

In this article, we will highlight the value of surveillance programs in enhancing our understanding of the trends of antimicrobial resistance both in systemic as well as ocular infections, and thereby highlighting the need for cooperative global and regional efforts not only for systemic infections but also for ocular or site-specific infections.

\section{Antimicrobial Surveillance}

Realizing the seriousness of the situation, in 1998, the World Health Assembly adopted a resolution urging Member States to take action against AMR. In 2001, World Health Organization (WHO) published the "WHO global strategy on containment of antimicrobial resistance" containing a comprehensive set of recommendations to enable member countries to formulate and implement national policies to curtail AMR [16]. The strategy recommended the surveillance of AMR as an essential part of the action plan, in the hope that the knowledge about the emergence and spread of resistant pathogens will help develop effective strategies for the prevention or control of the resistance problem.

After the World Health Assembly resolution, AMR surveillance systems have been instituted in many countries, at regional, national and supranational levels. Activities undertaken by these surveillance networks vary in their scope and magnitude: some focus on specific species and a small number of antimicrobial agents, while others are far more inclusive. Examples of some of the supranational surveillance systems developed in collaboration with WHO are: Integrated Disease Surveillance and Response (IDSR) in Africa; Red LatinoAmericana De Vigilancia De La Resistancia A Los Animicrobianos (ReLAVRA) in Canada, USA and South American nations; Antimicrobial resistance in the Mediterranean (ARMed); European Antimicrobial Resistance Surveillance Network (EARS-net); and Asian Network for Surveillance of Resistant Pathogens (ANSORP). To facilitate the gathering of comparable data from across the world, the WHO collaborating centre for surveillance of antimicrobial resistance developed WHONET, a Microsoft windows-based software to enter AMR data from individual patient samples manually or to capture data from automated laboratory systems [17]. The system provides a standardised format for data collection that can then be analysed at a local level or across one or more regions.

Apart from the supranational programmes supported by the World Health Organization, a number of national and regional surveillance activities have been conducted. Examples of such activities are: The National Healthcare Safety Network (NHSN), Prospective Resistant Organism Tracking and Epidemiology for the Ketolide Telithromycin (PROTEKT), SENTRY Antimicrobial Surveillance Program, European Antimicrobial Resistance Surveillance System (EARSS), Study for Monitoring Antimicrobial Resistance Trends (SMART), The Surveillance Network (TSN), and Tracking Resistance in the United States Today (TRUST) [18-24].

Such surveillance programmes have helped generate information on (1) trends of pathogens and AMR including regional variations and emergence of combined resistance, (2) conduct audits on antibiotic uses and study the relationship between antibiotic use and resistance,and (3) assessment of outcomes of national and regional interventions.

\section{Magnitude of AMR Assessed by Surveillance Studies for Systemic Infections}

As per EARS-net, the resistance of $S$. aureus isolates to methicillin (MRSA) in 2011 ranged from $0.3 \%$ in Norway to as high as $54.6 \%$ in Portugal. In the same year, the resistance of Escherichia coli isolates to third generation cephalosporins ranged from $3 \%$ in Sweden to $36.2 \%$ in Cyprus, while the resistance of Pseudomonas aeruginosa to fluoroquinolones varied from $5.4 \%$ in Norway to $58.7 \%$ in Slovakia [25]. The surveillance reports published in March 2011 and November 2012 suggest that the levels of resistance in the Gram-positive pathogens (Streptococcus pneumonia, S. aureus, Enterococcus faecium and E. faecalis) tend to be stabilising, or even decreasing in some countries, but there is a general increase of antimicrobial resistance in the Gram-negative pathogens (E. coli, Klebsiella pneumonia and $P$. aeruginosa) across Europe. In the period from 2002 to 2009, while the proportion of $E$. coli resistant to third generation cephalosporins increased from 1.7 to $8 \%$, the proportion of MRSA decreased from 21.5 to $19.7 \%$ [26•*]. The more serious trends were significant increases in combined resistance (defined as resistance to two, three or four antimicrobial classes) in E. coli. The proportion of $E$. coli resistant to two antibiotic classes increased from 7.8 to $12.4 \%$; to three antibiotic classes it increased from 3.1 to $6.7 \%$; and to four antibiotics it increased from 0.6 to $3.4 \%$. In contrast, the proportion of E. coli susceptible to all four antimicrobial classes decreased from $51.4 \%$ in 2002 to $41.7 \%$ in 2009 [26••].

Almost similar trends are seen in the United States. A laboratory-based surveillance of antimicrobial resistance patterns among $S$. aureus in 2005 showed MRSA rates of 59.2, 55 and $47.9 \%$ among non-ICU, ICU and out-patients, respectively [27]. Further, the prevalence of MRSA was showing rising trends. However, a more recent publication describing S. aureus infections from 2005 through 2010 
showed declining trends of MRSA during this period, similar to what have been reported from Europe [28•]. In contrast to Gram-positive organisms, the Gram-negative bacteria showed increased resistance to various antibiotics even in the USA. The antimicrobial susceptibility test results obtained from TSN database for urinary $E$. coli isolates obtained from 2000 to 2010 showed greatest increase in resistance for ciprofloxacin (3\% in 2000 to $17.1 \%$ in 2010) and trimethoprim-sulfamethoxazole (17.9-24.2\%), whereas ceftriaxone showed minimal change [29॰]. Analysis of resistance trends for key cell envelope-active antibacterial agents among ESKAPE pathogens showed that, between 2007 to 2011, Acinetobacter baumannii resistance to imipenem increased from 23.9 to $34.3 \%$ and to piperacillintazobactam increased from 37 to $49.7 \%$. During the same time period, $K$. pneumonia resistance to imipenem increased from 0.8 to $3.8 \%$ [30॰]. Since beta-lactams are a cornerstone of anti-infective therapy, the authors suggested close monitoring of the resistance trends.

Data from integrated surveillance brought out another important aspect of AMR: there is a clear association between antibiotic use and AMR. The data obtained from the European Surveillance of Antimicrobial Consumption and the European Antimicrobial Resistance Surveillance System correlating the use of antimicrobial agents in ambulatory care and the resistance trends of 2 major pathogens, S. pneumoniae and E. coli, clearly suggest that variation of consumption coincides with the occurrence of resistance at the country level [31]. A 9-year study from 1999 to 2007, undertaken in the US covering over 300 laboratories, demonstrated that the seasonal trends in consumption of antibiotics matched with corresponding increases or decreases in certain antimicrobial resistance patterns [32]. In 1998, Austin et al. [33] performed an analysis of the influence of the selective pressure imposed by the volume of drug use on temporal changes in resistance using population genetic methods and epidemiological observations. The analyses indicated that the time scale for emergence of resistance under a constant selective pressure is typically much shorter than the decay time after cessation or decline in the volume of drug use, and that significant reductions in resistance requires equally significant reductions in drug consumption [33]. Therefore, one needs to be aware of the fact that the impact of reducing antibiotic use on the reduction of resistance rates is complex, highlighting the difficulties involved in reversing resistance once it has become established.

\section{Antimicrobial Surveillance in Ophthalmology}

A review of ophthalmic literature suggests that while antimicrobial resistance is not a new phenomenon in ophthalmology, most publications on trends of antimicrobial resistance among ocular isolates have been generated by the experience of individual laboratories or institutions [34-41]. It is only in the last decade that a few large multicentre surveillance studies focussing on AMR among ocular isolates have been published.

First such surveillance study was a review of The Surveillance Network (TSN) data on ocular isolates of $S$. aureus submitted from January 2000 to December 2005 [42]. The study showed that the proportion of ocular infections caused by methicillin-resistant $S$. aureus increased from $29.5 \%$ in 2000 to $41.6 \%$ in 2005. The data also showed that the MRSA ocular isolates were resistant to 3 or more classes of antibiotics including fluoroquinolones, a property similar to the systemic isolates of MRSA.

Another surveillance study is Ocular Tracking Resistance in the U.S. Today (Ocular TRUST). The Ocular TRUST annually evaluates in vitro antimicrobial susceptibility of S. aureus, S. pneumoniae, and Haemophilus influenzae to a variety of commonly used ophthalmic antibiotics including fluoroquinolones, aminoglycosides, penicillins, macrolids, polymyxin B and trimethoprim at an independent central laboratory. The first report of the Ocular TRUST was published in 2008 [43] and comprised two sections: the first was a prospective surveillance study conducted from October 2005 through June 2006 wherein 7 eye hospitals and 28 community hospitals from 19 states submitted ocular isolates, and the second section contained archived ocular isolates submitted to the longitudinal surveillance program (TRUST) between 1999 and 2006, which were assessed using methodology identical to that of the prospective surveillance study. The data showed that the susceptibility of MSSA for the fluoroquinolones was 79.9 to $81.1 \%$ and that of MRSA was $15.2 \%$. MRSA isolates also exhibited high level of resistance to other classes of antibiotics except trimethoprim that had high activity against MRSA. All S. pneumoniae isolates were susceptible to fluoroquinolones except ciprofloxacin to which some level $(10.2 \%)$ of resistance was noticed. Haemophilus influenzae isolates were $100 \%$ susceptible to all tested agents except trimethoprim. The data from archived isolates showed similar findings as the Ocular TRUST 1 data. The authors concluded that, despite widespread use of fluoroquinolones as systemic therapy, ocular isolates are maintaining high levels of susceptibility to this class of antibiotics. The authors, therefore, supported the use of various fluoroquinolones for prophylaxis as well as treatment of ocular infections. However, the authors advised the consideration of alternatives to fluoroquinolones if MRSA is a suspected pathogen.

More recent ophthalmic surveillance study is the Antibiotic Resistance Monitoring in Ocular Microorganisms (ARMOR) 2009 [44••]. The study prospectively evaluated 
antimicrobial susceptibility of $S$. aureus, coagulase-negative Staphylococci, S. pneumoniae, $H$. influenzae, and $P$. aeruginosa isolated from cases of ocular infections. Thirtyfour institutions across the United States participated in the study and submitted isolates to a central laboratory for further testing. Minimum inhibitory concentrations for various antibiotics were determined by microbroth dilution.

The data from this study showed a no increase or a marginal reduction of MRSA as compared to data of 2005 from TSN (39 vs. 41.6\%). These prevalence trends of MRSA among ocular infections were similar to trends in systemic infections. The second significant finding of this study was the activity of fluoroquinolones against Staphylococcus. Fluoroquinolones showed significant differences in their activity against all Staphyloccoci, with basifloxacin being the most active antibiotics followed by moxifloxacin and ciprofloxacin; a remarkable shift compared to the observations made in the Ouclar TRUST study. It can be argued that the highest activity of basifloxacin is because of the non-availability of the antibiotic for systemic uses, as well as the relatively recent introduction for ophthalmology, again supporting the hypothesis that the level of AMR correlates with the level of the consumption of antibiotics.

The third observation of the ARMOR 2009 data that has important clinical applications was the occurrence of nonsusceptibility among $P$. aeruginosa isolates to third generation cephalosporins, fluoroquinolones and carbapenems. The prevalence of resistance ranged from $7 \%$ for ceftazidime and tobramycin to 11 and $13 \%$ for ciprofloxacin and imipenem, respectively. Further, the ciprofloxacin-resistant isolates were found to be resistant to other classes of antibiotics, indicating the emergence of multidrug-resistant Gram-negative infections in ophthalmology. With only $36.0 \%$ of all S. aureus and $21.5 \%$ of all CNS isolates being susceptible to the entire drug classes tested, the emergence of multidrug resistance even in $P$. aeruginosa is pointing to an impending threat in the management of infectious diseases of the eye.

It can be reasonably concluded that the organisms causing ocular infections are following similar trends as the organisms causing other systemic infections. In fact, it is often argued that the resistance among ocular isolates is primarily due to the inappropriate use of antibiotics for systemic infections, as well as their use in agriculture and by the veterinary profession. However, one must be aware of the fact that there is evidence that points towards the role of injudicious and prolonged use of topical antibiotics in promoting drug resistance [45•, 46••, 47, 48].

This review clearly highlights that the data generated through various surveillance programmes has been of immense value in enhancing our understanding of the epidemiology of AMR, both in systemic as well as ophthalmic infections. The MRSA have started showing a declining trend, but a worrisome trend is the increasing resistance among the Enterobacteriaceae group of organisms. More worrisome is the emergence of multidrugresistant pathogens. The surveillance studies have also established that there is a direct correlation between antimicrobial consumption and the level of resistance, and that the reversal of resistance on the cessation of the use of antibiotics is slow. All these conclusions from various surveillance studies should help health authorities to design policies for containing AMR.

However, one must be careful in generalising the conclusions from these studies because almost all the current literature on antibiotic resistance among both systemic and ocular infections came primarily from the US or Europe, and thus focus on organisms more prevalent in the West. Even the three ocular surveillance studies were carried out on bacterial isolates from the US. Since AMR is largely dependent on regional or national medical practice patterns, it will be important for each region and nation to design local surveillance studies.

Realizing that there is no consistent and comparative data on antimicrobial profiles and antibiotic resistance in Asia, the Asia Cornea Society proposed a prospective multi-center study known as the Asia Cornea Society Infectious Keratitis Study (ACSIKS). Major aims of the ACSIKS are: to establish the prevalence of eye infections in developed and developing Asian countries, determine the causative risk factors, and the range of organisms as well as antimicrobial resistance patterns. Twelve centres from 8 countries from the Asia Pacific region are participating in the study. All participating centres are following a common microbiology protocol including the set of antibiotics for susceptibility tests. The ACSIKS is expected to provide useful information on AMR patterns among ocular isolates from this region. Similar efforts are needed in other geographical areas including Africa, Middle East and South America. Further, the ophthalmology programs must have a link or be networked with the programmes focused on systemic infections. This approach will help us better understand AMR trends compared to systemic trends.

\section{Conclusion}

Antimicrobial resistance is a serious problem not only for systemic infections but also in ophthalmology. The resistance among Enterobacteriaceae and multidrug resistance are emerging threats both for systemic and ocular infections. Since there is a clear association between the use of antibiotics and the resistance, we need strong antibiotic policies for containing this challenge. There is also an urgent need to find new drugs or ways to treat diseases caused by highly resistant microorganisms. 
Disclosure Prashant Garg has board membership with Bausch \& Lomb and NovBay, is a consultant to Alcon and Allergan, and has received travel/accommodation/meeting expenses from Allergan, Bausch \& Lomb, and Alcon. Aravind Roy declares no conflict of interest.

Compliance with Ethics Guidelines This article does not contain any studies with human or animal subjects performed by any of the authors.

Financial Disclosure None of the authors have any financial interest in the subject matter discussed in the paper.

\section{References}

Papers of particular interest, published recently, have been highlighted as:

- Of importance

- Of major importance

1. Ashley DJ, Brindle MJ. Penicillin resistance in staphylococci isolated in a casualty department. J Clin Pathol. 1960;13:336-8.

2. Bolton H, Catchpole BN, Jepson RP. Penicillin in pulp-space infections of the finger. Lancet. 1947;2(6478):608-10.

3. Birnstingl MA, Shooter RA, Hunt MF. Sensitivity to five antibiotics of strains of Staph. pyogenes isolated from outpatients. $\mathrm{Br}$ Med J. 1952;2(4778):253-4.

4. Jessen O, Rosendal K, Bulow P, Faber V, Eriksen KR. Changing staphylococci and staphylococcal infections. A ten-year study of bacteria and cases of bacteremia. N Engl J Med. 1969;281(12): $627-35$

5. Hughes GB, Chidi CC, Macon WL. Staphylococci in communityacquired infections: increased resistance to penicillin. Ann Surg. 1976;183(4):355-7.

6. - Paphitou NI. Antimicrobial resistance: action to combat the rising microbial challenges. Int J Antimicrob Agents. 2013;42 Suppl:S25-28. This review article gives an overview of the problem of antimicrobial resistance and the multifaceted approach required to contain it.

7. McConnell J. WHO announce strategy to combat antimicrobial resistance. Lancet Infect Dis. 2001;1(3):140.

8. Zarocostas J. Urgent efforts needed globally to combat the spread of antimicrobial resistance, says WHO. BMJ. 2011;342:d2205.

9. Simonsen GS, Tapsall JW, Allegranzi B, Talbot EA, Lazzari S. The antimicrobial resistance containment and surveillance approach-a public health tool. Bull World Health Organ. 2004;82(12):928-34.

10. Kardas-Sloma L, Boelle PY, Opatowski L, et al. Impact of antibiotic exposure patterns on selection of community-associated methicillin-resistant Staphylococcus aureus in hospital settings. Antimicrob Agents Chemother. 2011;55(10):4888-95.

11. Spellberg B, Powers JH, Brass EP, Miller LG, Edwards JE Jr. Trends in antimicrobial drug development: implications for the future. Clin Infect Dis. 2004;38(9):1279-86.

12. Braine T. Race against time to develop new antibiotics. Bull World Health Organ. 2011;89(2):88-9.

13. Cohen ML. Epidemiology of drug resistance: implications for a post-antimicrobial era. Science. 1992;257(5073):1050-5.

14. Birnbaum D. Antimicrobial resistance: a deadly burden no country can afford to ignore. Can Commun Dis Rep. 2003;29(18):157-64.

15. de Kraker ME, Davey PG, Grundmann H. Mortality and hospital stay associated with resistant Staphylococcus aureus and
Escherichia coli bacteremia: estimating the burden of antibiotic resistance in Europe. PLoS Med. 2011;8(10):e1001104.

16. WHO Global Strategy for Containment of Antimicrobial Resistance. Geneva: World Health Organization; 2001. Available on http://www.who.int/csr/resources/publications/drugresist/en/ EGlobal_Strat.pdf. Accessed 9 Jan 2012.

17. WHONET available at http://www.who.int/drugresistance/ whonetsoftware/en/.

18. Intensive Care Antimicrobial Resistance Epidemiology (ICARE). Surveillance Report, data summary from January 1996 through December 1997: a report from the National Nosocomial Infections Surveillance (NNIS) System. Am J Infect Control. 1999;27(3):279-84.

19. Thornsberry C, Sahm DF, Kelly LJ, et al. Regional trends in antimicrobial resistance among clinical isolates of Streptococcus pneumoniae, Haemophilus influenzae, and Moraxella catarrhalis in the United States: results from the TRUST Surveillance Program, 1999-2000. Clin Infect Dis. 2002;34(Suppl 1):S4-16.

20. Jones RN. Global epidemiology of antimicrobial resistance among community-acquired and nosocomial pathogens: a fiveyear summary from the SENTRY Antimicrobial Surveillance Program (1997-2001). Semin Respir Crit Care Med. 2003;24(1): 121-34.

21. Marchese A, Schito GC. Recent findings from multinational resistance surveys: are we 'PROTEKTed' from resistance? Int J Antimicrob Agents. 2007;29(Suppl 1):S2-5.

22. Bronzwaer SL, Cars O, Buchholz U, et al. A European study on the relationship between antimicrobial use and antimicrobial resistance. Emerg Infect Dis. 2002;8(3):278-82.

23. Sheng WH, Badal RE, Hsueh PR. Distribution of extendedspectrum beta-lactamases, AmpC beta-lactamases, and carbapenemases among enterobacteriaceae isolates causing intraabdominal infections in the Asia-Pacific region: results of the study for Monitoring Antimicrobial Resistance Trends (SMART). Antimicrob Agents Chemother. 2013;57(7):2981-8.

24. Karchmer AW. Increased antibiotic resistance in respiratory tract pathogens: PROTEKT US-an update. Clin Infect Dis. 2004; 39(Suppl 3):S142-50.

25. Antimicrobial resistance surveillance in Europe. 2011. Available at http://www.ecdc.europa.eu/en/publications/surveillance_reports.

26. •• Gagliotti C, Balode A, Baquero F, et al. Escherichia coli and Staphylococcus aureus: bad news and good news from the European Antimicrobial Resistance Surveillance Network (EARS-Net, formerly EARSS), 2002 to 2009. Euro Surveill. 2011;16(11). This study describes the trends of antimicrobial susceptibility patterns and occurrence of invasive infections in Europe by two most common pathogens-Escherichia coli and Staphylococcus aureus in the period from 2002 to 2009.

27. Styers D, Sheehan DJ, Hogan P, Sahm DF. Laboratory-based surveillance of current antimicrobial resistance patterns and trends among Staphylococcus aureus: 2005 status in the United States. Ann Clin Microbiol Antimicrob. 2006;5:2.

28. - Landrum ML, Neumann C, Cook C, et al. Epidemiology of Staphylococcus aureus blood and skin and soft tissue infections in the US military health system, 2005-2010. JAMA. 2012;308(1):50-59. This report demonstrated the declining trends of both community-onset and hospital-onset MRSA bacteremia as well as community-onset skin and soft tissue infections.

29. - Sanchez GV, Master RN, Karlowsky JA, Bordon JM. In vitro antimicrobial resistance of urinary Escherichia coli isolates among U.S. outpatients from 2000 to 2010. Antimicrob Agents Chemother. 2012;56(4):2181-83. This study examined resistance data of Escherichia coli isolates obtained from urine samples of U.S. outpatients between 2000 and 2010 and found a substantial increase in resistance to ciprofloxacin and trimethoprimsulfamethoxazole. 
30. - Master RN, Deane J, Opiela C, Sahm DF. Recent trends in resistance to cell envelope-active antibacterial agents among key bacterial pathogens. Ann N Y Acad Sci. 2013;1277:1-7. This study evaluated resistant trends for key cell envelope-active drugs particularly beta lactams among ESKAPE pathogens.

31. van de Sande-Bruinsma N, Grundmann H, Verloo D, et al. Antimicrobial drug use and resistance in Europe. Emerg Infect Dis. 2008;14(11):1722-30.

32. Bergman M, Nyberg ST, Huovinen P, Paakkari P, Hakanen AJ. Association between antimicrobial consumption and resistance in Escherichia coli. Antimicrob Agents Chemother. 2009;53(3):912-7.

33. Austin DJ, Kristinsson KG, Anderson RM. The relationship between the volume of antimicrobial consumption in human communities and the frequency of resistance. Proc Natl Acad Sci USA. 1999;96(3):1152-6.

34. Jayahar Bharathi M, Ramakrishnan R, Ramesh S, Murugan N. Extended-spectrum beta-lactamase-mediated resistance among bacterial isolates recovered from ocular infections. Ophthalmic Res. 2012;47(1):52-6.

35. Shalchi Z, Gurbaxani A, Baker M, Nash J. Antibiotic resistance in microbial keratitis: ten-year experience of corneal scrapes in the United Kingdom. Ophthalmology. 2011;118(11):2161-5.

36. Mohammadpour M, Mohajernezhadfard Z, Khodabande A, Vahedi P. Antibiotic susceptibility patterns of pseudomonas corneal ulcers in contact lens wearers. Middle East Afr J Ophthalmol. 2011;18(3):228-31.

37. Kim SJ, Toma HS. Antimicrobial resistance and ophthalmic antibiotics: 1-year results of a longitudinal controlled study of patients undergoing intravitreal injections. Arch Ophthalmol. 2011;129(9):1180-8.

38. Hsiao CH, Chuang CC, Tan HY, Ma DH, Lin KK, Chang CJ, Huang YC. Methicillin-resistant Staphylococcus aureus ocular infection: a 10-year hospital-based study. Ophthalmology. 2012;119(3):522-7.

39. Shimizu Y, Toshida H, Honda R, Matsui A, Ohta T, Asada Y, Murakami A. Prevalence of drug resistance and culture-positive rate among microorganisms isolated from patients with ocular infections over a 4-year period. Clin Ophthalmol. 2013;7:695-702.

40. Lichtinger A, Yeung SN, Kim P, et al. Shifting trends in bacterial keratitis in Toronto: an 11-year review. Ophthalmology. 2012; 119(9):1785-90.
41. Zhou AW, Lee MC, Rudnisky CJ. Ocular microbiology trends in Edmonton, Alberta: a 10-year review. Can J Ophthalmol. 2012; 47(3):301-4.

42. Asbell PA, Sahm DF, Shaw M, Draghi DC, Brown NP. Increasing prevalence of methicillin resistance in serious ocular infections caused by Staphylococcus aureus in the United States: 2000 to 2005. J Cataract Refract Surg. 2008;34(5):814-8.

43. Asbell PA, Colby KA, Deng S, et al. Ocular TRUST: nationwide antimicrobial susceptibility patterns in ocular isolates. Am J Ophthalmol. 2008;145(6):951-8.

44. •• Haas W, Pillar CM, Torres M, Morris TW, Sahm DF. Monitoring antibiotic resistance in ocular microorganisms: results from the Antibiotic Resistance Monitoring in Ocular micRorganisms (ARMOR) 2009 surveillance study. Am J Ophthalmol. 2011; 152(4):567-74 e563. This laboratory-based surveillance study determined the antibacterial susceptibility profile of bacterial pathogens from ocular infections against a variety of antibacterial agents. The samples were collected prospectively by 34 institutions in the US. The study showed declining activity of fluoroquinolones and the emergence of multidrug-resistant infections in ophthalmology.

45. - Fintelmann RE, Hoskins EN, Lietman TM, et al. Topical fluoroquinolone use as a risk factor for in vitro fluoroquinolone resistance in ocular cultures. Arch Ophthalmol. 2011;129(4):399-402. Contrary to popular belief, this study demonstrated that the use of topical antibiotics is associated with increased resistance among ocular isolates.

46. •• Yin VT, Weisbrod DJ, Eng KT, et al. Antibiotic resistance of ocular surface flora with repeated use of a topical antibiotic after intravitreal injection. JAMA Ophthalmol. 2013;131(4):456-61. This study demonstrated that the repeated use of topical antibiotics for prophylaxis against infection can result in a significant increase in antimicrobial resistance of ocular surface flora.

47. Ray KJ, Prajna L, Srinivasan M, et al. Fluoroquinolone treatment and susceptibility of isolates from bacterial keratitis. JAMA Ophthalmol. 2013;131(3):310-3.

48. Dave SB, Toma HS, Kim SJ. Ophthalmic antibiotic use and multidrug-resistant Staphylococcus epidermidis: a controlled, longitudinal study. Ophthalmology. 2011;118(10):2035-40. 